\title{
Ground experiment on the instability of buoyant-thermocapillary convection in large-scale liquid bridge with large Prandtl number
}

\author{
Jia Wang, Di Wu, Li Duan, Qi Kang*

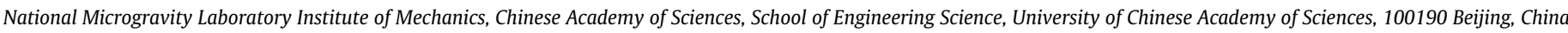

\section{A R T I C L E I N F O}

Article history:

Received 12 July 2016

Received in revised form 19 December 2016

Accepted 25 December 2016

Available online 25 January 2017

\section{Keywords:}

Large-scale liquid bridge

Marangoni convection

Transition process

Hydrothermal wave

Volume ratio

\begin{abstract}
A B S T R A C T
In order to cooperate with the Chinese TG-2 space experiment project, this paper studies the flow structure and critical conditions at the onset of transition and nonlinear regimes of bouyant-thermocapillary convection in large-scale liquid bridge with large Prandtl number under normal gravity. The surface temperature distribution is obtained by means of thermal infrared camera, to study the temperature oscillation, temporal-spatial analysis and modal structures of the temperature field. In addition, the fluid velocity field is measured by Particle Image Velocimetry, to study the internal flow field structure and flow characteristics in the transition process of the liquid bridge. It is found that the critical value of the buoyant-thermocapillary convection in the half-zone liquid bridge can be affected by geometric parameters. Under large Prandtl number conditions, the critical temperature difference will change nonlinearly with the volume ratio, and the convection will transit from steady flow to a sequence of instabilities. In addition, various wave patterns will appear with increasing Marangoni number, and with further increased temperature difference a chaos state will emerge.
\end{abstract}

(c) 2017 Elsevier Ltd. All rights reserved.

\section{Introduction}

Marangoni convection is the fluid motion driven by surface tension differences caused by thermal gradients along a liquid-gas liquid interface [1], which becomes of particular importance in flow systems such as droplets, bubbles, liquid containers [2] and liquid bridges.

A half floating zone liquid bridge (hereafter referred to as an LB), which is some liquid limited to two coaxial rods by surface tension, has been proposed to produce high-quality single crystals of high purity. Due to the fact that the surface tension gradient is decided negatively by the temperature, the driving force is directed from the cooler end to the hotter end of the gas-liquid interface. Through the joint effects of gravity and surface tension, Marangoni convection is generated in the zone. The flow driven by the temperature difference between the disks, namely $\Delta T$, is directed from the upper heated disk toward the lower cooled disk along the free surface, after which it returns back inside the liquid. It is well known that when the $\Delta T$ exceeds the critical value $\Delta T_{c}$, the Marangoni convection in the bridge will transform from a steady, axisymmetric state to an oscillatory, non-axisymmetric one. The appearance of such an unsteady flow can lead to the severe

\footnotetext{
* Corresponding author.

E-mail address: kq@imech.ac.cn (Q. Kang).
}

deterioration of the quality in the grown crystals. The mechanisms of instability have universal characteristics in many fields. This is of general interest, far beyond its direct applications in engineering, thus the LB problem has been considered as a paradigmatic case of nonlinear dynamics in essentially nonparallel flows. Consequently, a large number of studies have been conducted regarding the instability mechanisms in LBs.

The onset of such instability is the function of many parameters, such as geometric parameters, Prandtl (hereafter referred to as $\mathrm{Pr}$ ) number, and air natural convection. These influences can be shown by the critical point, frequency, flow mode, and so on. Levenstam et al. [3] studied the transition process with small Pr number by means of direct numerical simulation, and pointed out that there were two transitions with the increasing $\Delta T$. This conclusion was later confirmed under large $\operatorname{Pr}$ number conditions by Tang et al. [4], but the process was shown to have been affected by geometric parameters. Li et al $[5,6]$ and Chen et al [7] respectively numerically studied the effects of these parameters on the critical point in the case of small and large Pr numbers. Xu et al. [8] and Zeng et al. [9] found that these parameters and $\operatorname{Pr}$ number also partially determined the flow mode.

It should be noted that the oscillatory flow also can be determined by the initial perturbations introduced to the dynamical system [10]. Monti et al. [11] reported that the numerical calculations could not comprehensively simulate the experimental 
conditions. In terrestrial gravity experiments, in order to weaken the effect of gravity, the typical small scale LBs in the ground experiments are generally $4-5 \mathrm{~mm}$ in diameter [1]. The relationship between the geometric parameters and critical conditions has been previously provided [12-14]. Frank et al. [15] found that there were pulsating waves and rotating waves in LB. Schwabe et al. [16] pointed out these two types of waves could co-exist in the transition process. The demand for large-scale (i.e. diameter greater than $5 \mathrm{~mm}$ ) crystals has grown with the advancement of technology development. As KIBO reported, the difference in size of LBs ( $30 \mathrm{~mm}$ vs $50 \mathrm{~mm}$ in disk diameter) could cause the results to differ [17]. Therefore, small-scale liquid bridges were shown to be unsuitable for effectively simulating large-scale crystal growth.

Japan $[18,19]$ has conducted the study of the instability of largescale LB on the International Space Station (ISS) due to the reduction of gravity in space. Considering the high costs and limited number of opportunities for space experiments, performing the study of large-scale LB on the ground in advance is particularly important. To coincide with the Chinese TG- 2 space experiment project, matching experiment research under normal gravity has been launched, in which the bridge is $20 \mathrm{~mm}$ in diameter.

In the study of the mechanism of thermocapillary oscillation of LBs, most theories hold that the oscillation is due to the internal instability induced by thermocapillary convection, thus the various unstable flow modes should be studied using unstable processing methods. Another view is that the oscillation in LB is not due to the instability of the dynamic system, but that the large deformation of the free surface is caused by the interaction of internal flow and thermal process in thermocapillary convection, thereby maintaining the oscillation. Due to the non-linear characteristics of the flow in LBs, a series of characteristic parameters defining the free surface topography of LBs, and the initial conditions of the flow system, the effects of these on the structure of the oscillatory flow in LBs cannot be ignored.

Due to the fact that the thermocapillary convection of LBs is coupled with buoyancy and surface tension, the energy transmission promotes fluid flow, and in turn the fluid flow accelerates the spread of energy, so that the flow field structure in LBs becomes very complicated. To further reveal the flow mechanism, it is necessary to study the temperature field and velocity field of the thermocapillary convection in LBs. However, in most LB experiments, including the TG-2 space experiment, the study of the internal flow mechanism is limited to single point temperature measurement by thermocouples. Because of this, we have failed to directly observe the flow state such as the non-axisymmetry, oscillation, and temperature distribution of the flow field, which causes much inconvenience to the discrimination of the flow mode, let alone the measurement of velocity. In addition, the contact measurement and instrument accuracy may result in many restrictions in the experimental conditions, as well as considerable interference in the experimental results. Given this, in our experiment, aside from the thermocouple being used to measure the single point temperature, we also use the infrared thermal camera to directly observe the temperature distribution, flow mode, hydrothermal waves, and so on. In addition, the PIV (Particle Image Velocimetry) method was applied, a transparent sapphire upper disk was made, and through CCD we observed the movement of tracer particles in the cross-section, in order to better understand the flow of the liquid.

In the present paper, by observing and analyzing the temperature and velocity field of large scale LBs, we have obtained the critical conditions of the transition and basic flow discipline. At the same time, we have determined a relatively completed flow mode, and discussed the critical conditions and characteristics to a great degree of detail. In addition, the volume ratio effect and two transition processes of large scale LBs were verified for the first time on the ground.

\section{Experimental apparatus}

The half-zone LB is held between two coaxial rods heated differentially, for the purpose of the measurement of temperature distribution by means of infrared camera and velocity field by Particle Image Velocimetry (PIV) of thermo-convection. Rather than common bridge disks made of brass, we adopt specially designed disks, as illustrated in Fig. 1. The upper heated rod consists of transparent infrared glass which infrared light $\left(\lambda\right.$ infrared glass $\left.=11.7 \mathrm{Wm}^{-{ }^{\circ} \mathrm{C}-1}\right)$ can pass through, and the cooling brass rod is placed exactly beneath the heated one, separated by distance $H$. In addition, another heated transparent rod is made of sapphire with high thermal conductivity $\left(\lambda\right.$ sapphire $\left.=45.0 \mathrm{Wm}^{-1^{\circ} \mathrm{C}-1}\right)$ and resistance. These transparent upper rods allow us to have a top view of the velocity and temperature fields avoiding lossy measurement. Due to the fact that the thermal conductivity of sapphire and infrared glass are much higher than that of the working fluid-silicone oil, the radial temperature distribution is nearly constant, the LB model in this paper is equivalent to the brass one. Both rods are $20 \mathrm{~mm}$ in diameter, with a small edge to prevent liquid leakage, as leakage can greatly influence the liquid volume.

For the purpose of avoiding undesired buoyant convection, the lower rod is maintained at the ambient temperature, by means of semiconductor coolers. The heated plate can move in the $z$ axial direction, and a small range of aspect ratio $\operatorname{Ar}(=H / 2 \mathrm{R})$ can be realized in this large-scale LB due to gravity. Moreover, the volume ratio $\operatorname{Vr}\left(=V / V_{0}\right)$, which denotes the ratio of the liquid volume $V$ to the gap between the disks $\pi \mathrm{R}^{2} H, V_{0}$, can be realized by injection. The parametric ranges covered by the experiment are $0.15 \leqslant A r \leqslant 0.20$ and $0.54 \leqslant V r \leqslant 1.00$. To prevent the thermocouples from damaging the free surface, the $V r$ of the PIV is from 0.60 to 1.00 .

In the considered case of Japanese space experimental results and related numerical simulation [18,20], the temperature will oscillate when the Marangoni number exceeds about 20,000. For this reason, choosing silicone oil of 2cSt (Shin-Etsu Chemical Co., Ltd., KF-96L) [21] as the working liquid can offer a comparatively large Marangoni number, which the experimental conditions can achieve, the physical properties of which are shown in Table 1 . This makes it possible to observe the entire process of the convection from steady to oscillation in a single experiment. In order to weaken the influence of air convection on evaporation, we perform all of the experiments, except for the PIV measurement, in a sealed polymethyl methacrylate chamber, and measure the evaporation of silicone oil for each experiment.

To obtain an overview of the temperature field, a resolution of $320 \times 240$ pixels and thermal sensitivity smaller than $0.05^{\circ} \mathrm{C}$ US FLIR infrared camera (IR) with $4 \mathrm{x}$ macro lens $(10 \mu \mathrm{m})$ are used to observe the surface temperature distribution. The experiments are performed in a dark enclosed room, and the ambient temperature is kept at a constant temperature $\left(20^{\circ} \mathrm{C}\right)$, equipped with an infrared camera with appropriate emissivity and working distance.

The PIV system consists of a computer, CCD, laser and processing software. The CCD has a resolution of $640 \times 480$ pixels, and the sampling frequency can reach up to 260 fps. The sampling frequency of $140 \mathrm{fps}$ is adopted for the reason that the fluid velocity is not quite as fast. Silver hollow glasses microsphere of $10 \mu \mathrm{m}$ is chosen as the tracer particle. Due to the fact that the bridge itself is equal to a lens with different magnifications at the center and edge, the PIV results will produce considerable geometric distortions. Therefore, we illuminate the horizontal cross-section of the flow field at the maximum curvature of the liquid column by 


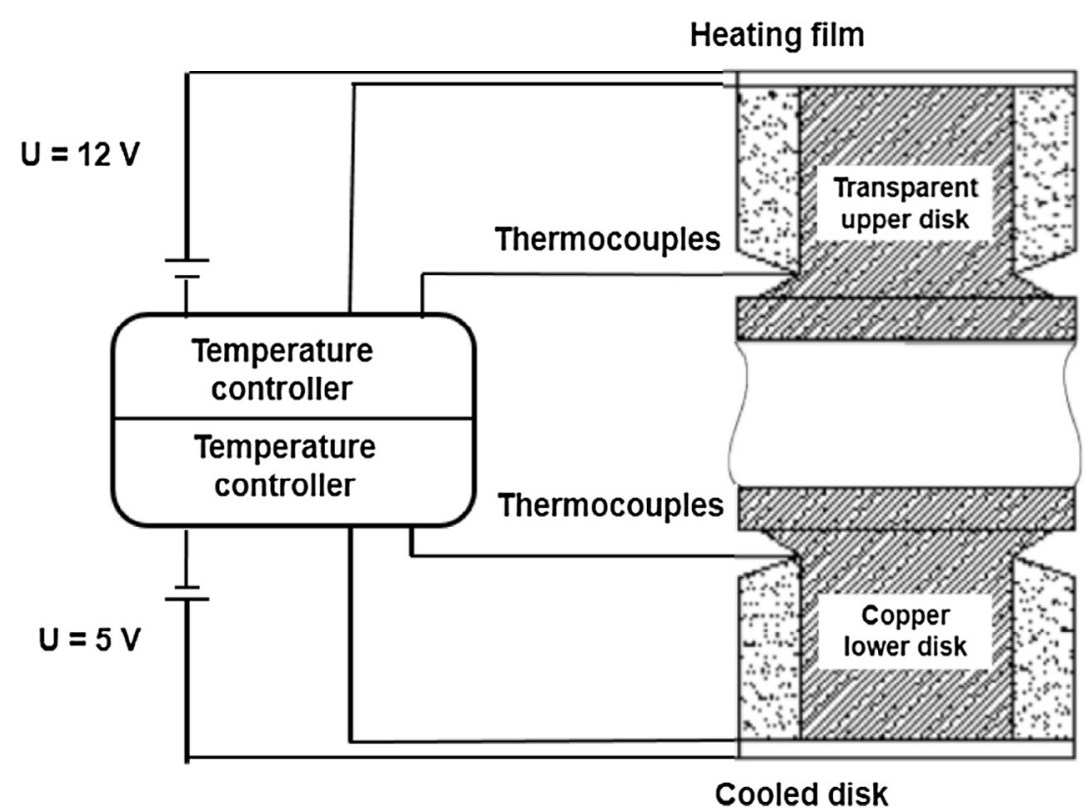

(a) Double road temperature control system

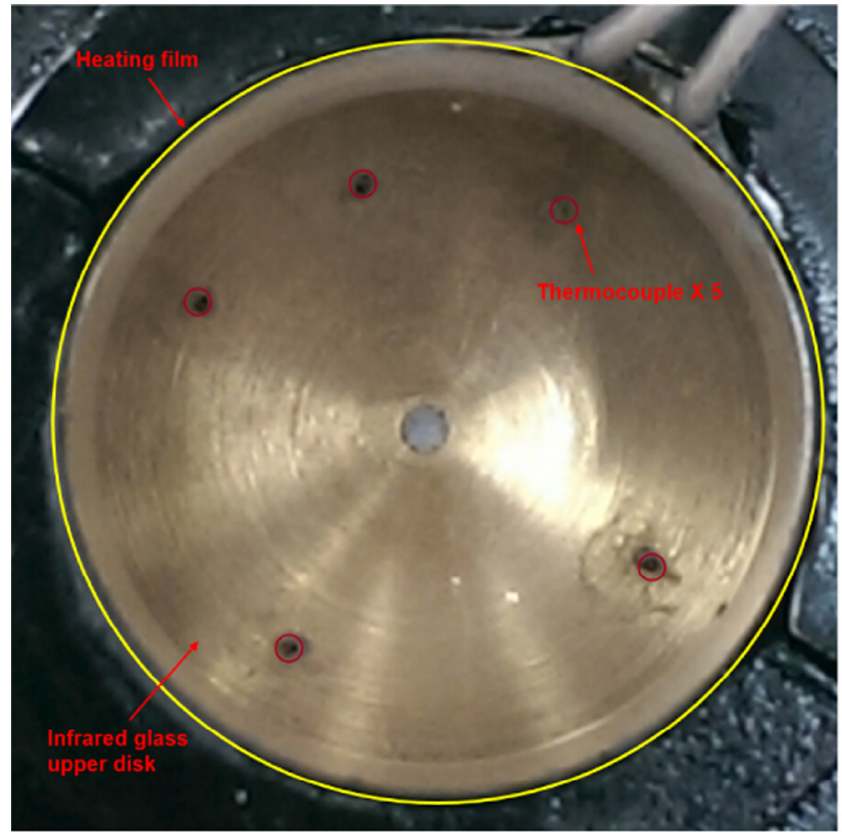

(b) Lateral view

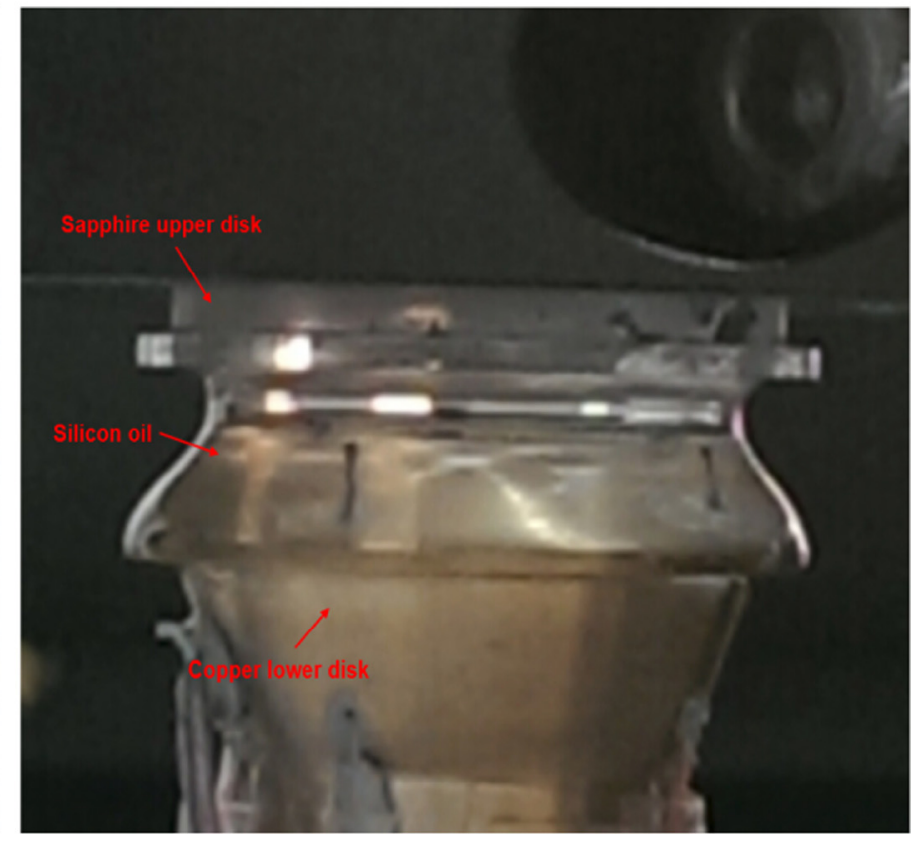

(c) Vertical view

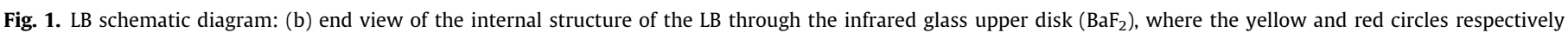

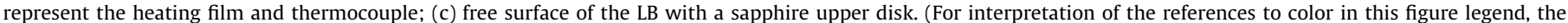
reader is referred to the web version of this article.)

Table 1

Physical properties of KF96-2 at working temperature.

\begin{tabular}{|c|c|c|c|c|c|c|c|}
\hline Silicon oil & $\gamma, \mathrm{m}^{2} / \mathrm{s}$ & $\rho, \mathrm{kg} / \mathrm{m}^{3}$ & $\beta, 1 /{ }^{\circ} \mathrm{C}$ & $\kappa, \mathrm{m}^{2} / \mathrm{s}$ & $\sigma, \mathrm{N} / \mathrm{m}$ & $\sigma_{\mathrm{T}}, \mathrm{N} /\left(\mathrm{m} \cdot{ }^{\circ} \mathrm{C}\right)$ & $\operatorname{Pr}$ \\
\hline $2 \mathrm{cSt}$ & $2.00 \times 10^{-6}$ & $8.7 \times 10^{2}$ & $1.24 \times 10^{-3}$ & $7.12 \times 10^{-8}$ & $1.83 \times 10^{-2}$ & $-7.15 \times 10^{-5}$ & 28.1 \\
\hline
\end{tabular}

means of a parallel sheet beam formed by a cylindrical lens, and capture the particle images above the upper rod by CCD. The velocity vector can be obtained by self-correlation calculations, so that the flow characteristics and vortex cell structures can be further studied.
The temperature acquisition system includes the computer, an Agilent 34970A 61/2 digital multimeter, and T-type thermocouples. There are seven thermocouples, two of which respectively collect the hot and cold end temperatures, and then report back to the control system for regulating the temperature difference, while 
the others are located at different azimuths of the same circumference (the diameter of which is $15 \mathrm{~mm}$ ) on the bottom rod to measure the single point temperature signals. From the temperature amplitude and phase differences, we can determine the flow field structures and flow patterns. The temperature measurement point is $2 \mathrm{~mm}$ higher above the bridge floor, and the holes are filled with thermal silica to avoid leakage (as shown in Fig. 1(c)). The maximum temperature difference among the five thermocouples is $0.15^{\circ} \mathrm{C}$ when the heater works without a carrier, and the median is selected as the measurement result. The sampling rate is $6.67 \mathrm{~Hz}$, which meets the experimental measurement requirements.

A ERUOTHERM 3504 temperature regulator is used for controlling the temperature by means of proportional integral differential (PID) algorithm, and the temperature stability is controlled within less than $0.01{ }^{\circ} \mathrm{C}$, thus the temperature oscillation measured is induced absolutely by the surface temperature oscillation. The entire experimental apparatus is shown in Fig. 2.

\section{Results and discussions}

\subsection{Parameter definition}

In our experiment, the applied temperature difference is increased from $0{ }^{\circ} \mathrm{C}$ to that of the oscillation at the rate of $0.3^{\circ} \mathrm{C}$ per minute, then step heating, and every step is maintained for $10 \mathrm{~min}$. To calculate $\Delta T_{\mathrm{c}}$ we utilize the fact that the amplitude of oscillating temperature grows as the square root of the distance from the bifurcation point [22], i.e. $A \sim\left(\Delta T-\Delta T_{\mathrm{c}}\right)^{1 / 2}$. By calculating the amplitude at several supercritical values of $\Delta T$ we can determine the critical one. In order to accomplish the transition procedure in a single experiment, several measurements at the subcritical values of $\Delta T$ are performed to obtain the onset. To facilitate understanding, $\Delta T_{\mathrm{c}}$ is non-dimensionalized into the critical Marangoni number, namely $M a_{\mathrm{c}}$.

It is important to note that the temperature dependence of the fluid viscosity could not be ignored in experiments with a large $\Delta T$. Using the empirical correlation formula, we estimate the average of the hot and cool ends ( $T_{\text {heat }}$ and $T_{\text {cool }}$ ) as the viscosity [21]:

$T=\left(T_{\text {heat }}+T_{\text {cool }}\right) / 2$

$\frac{v(T)}{v_{0}}=\exp \left(5.892 \frac{25-T}{273.15+T}\right)$

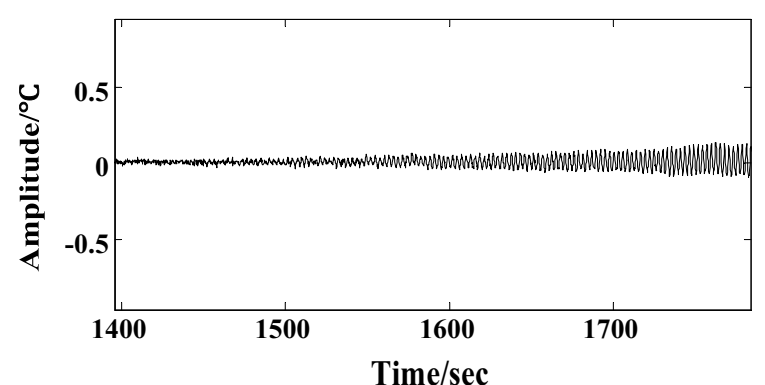

(a) Single point temperature with increasing temperature difference

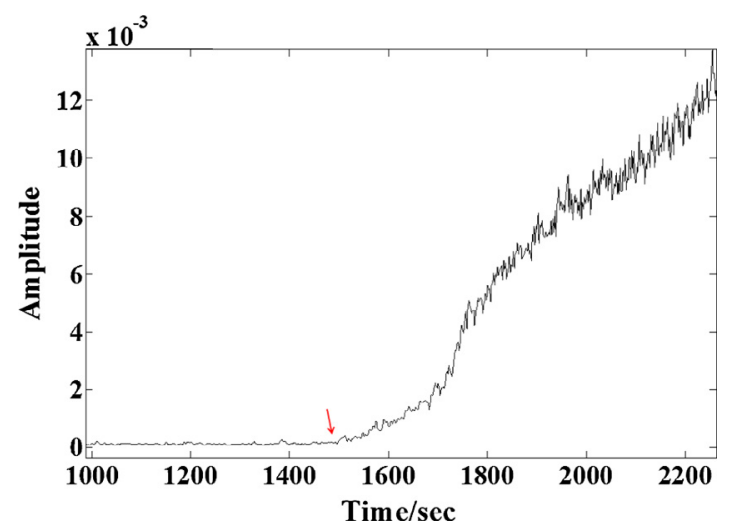

(b) The variance curve

Fig. 3. Judgment of the critical temperature: (a) the long-term trend of temperature signal is removed; (b) when the variances of several adjacent data windows are larger than a certain threshold value, the first numerical of the first window is regarded as the initial point (indicated by the red arrow).

Where $v_{0}$ and $T$ are the kinematic viscosity at $25^{\circ} \mathrm{C}$ and the ambient temperature, respectively. The Marangoni number can be defined as follows:

$M a=\frac{\left|\sigma_{T}\right| \Delta T H}{\rho v \alpha}$

The critical conditions are not the only purpose of this study. Another important question is how the dynamics of the flow change while one moves farther away from the critical points with

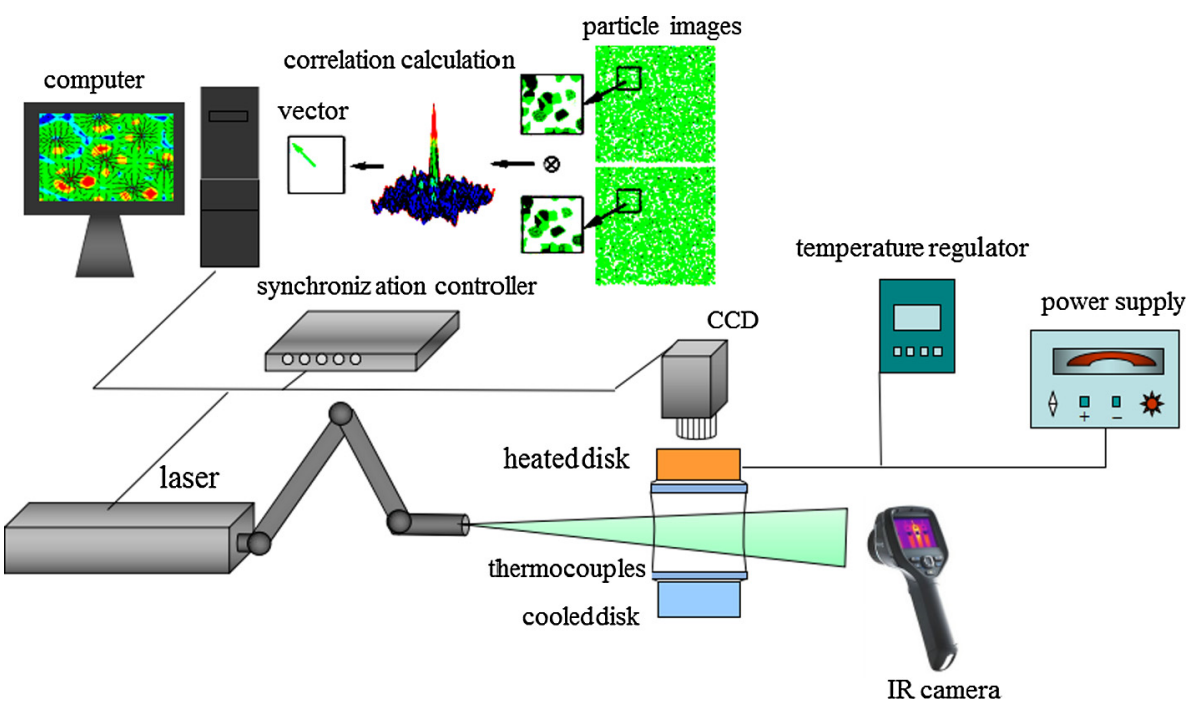

Fig. 2. Experimental setup. 
increasing $\Delta T$. Any significant changes in the dynamics are expected to be observed on the temperature field distribution. To trace the transitions occurring in the system, the temperature fields are analyzed using various methods. Fig. 4 summarizes the pseudo-color processing temperature field distribution performed for the top plane and free surface captured by IR, and the single point temperature oscillations recorded by thermocouples. To observe the effect of temperature difference on the instability, time evolution of wave propagation has also been performed by selecting a series of temperature signals on the circumference changing over time. The horizontal and vertical axes respectively represent the time and position, and the color shows the temperature amplitude.

As shown in Table 2, the flow structures can be classified into six regimes based on the ratio of the current Marangoni number to the critical one $\left(M a / M a_{\mathrm{c}}\right)$, judging from the temperature distribution and Fourier spectrum of the single temperature variation. The details of the experimental conditions for the top end surface are listed in Table 3.

\subsection{Modal structures transformation}

With a small Marangoni number, i.e. less than $0.8 M a_{\mathrm{c}}$, the temperature field in $\operatorname{Rg} 1$ demonstrates the 2D axisymmetric stationary solution without any fluctuation (as shown in Fig. 4(a)). The
Table 2

Flow regimes in the half-zone LB.

\begin{tabular}{lll}
\hline Regime & Flow pattern & $M a / M a_{\mathrm{c}}$ \\
\hline Rg1 & Steady axisymmetric & $0-0.8$ \\
Rg2 & Steady nonaxisymmetric & $0.8-1.0$ \\
Rg3 & Standing wave & $1.0-1.2$ \\
Rg4 & Traveling wave & $1.2-1.9$ \\
Rg5 & Local two waves separation & $1.9-2.1$ \\
Rg6 & Chaotic & 2.1 \\
\hline
\end{tabular}

Table 3

Experimental conditions of the typical flow regimes shown in Fig. 3.

\begin{tabular}{lll}
\hline Flow regime & $V r$ & $A r$ \\
\hline Rg1 & 0.91 & 0.20 \\
Rg2 & 0.86 & 0.17 \\
Rg3 & 0.62 & 0.15 \\
Rg4 & 0.62 & 0.15 \\
Rg5 & 0.85 & 0.20 \\
Rg6 & 0.85 & 0.20 \\
\hline
\end{tabular}

flow pattern is a circular in radial circumferential $(r-\varphi)$ plane. The center of the circle coincides with that of the upper rod, each point temperature in the field is the same everywhere, and the temperature curve is even like a horizon line. (a)
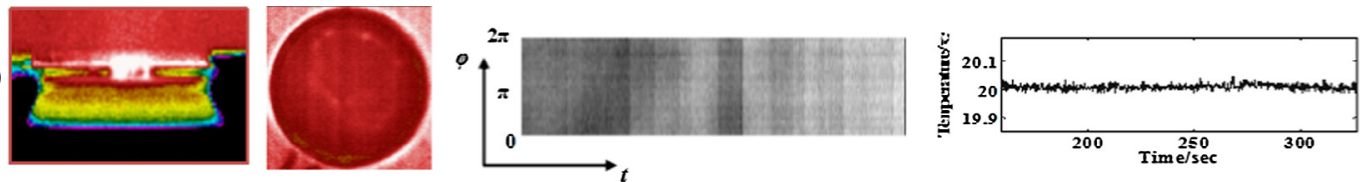

(b)
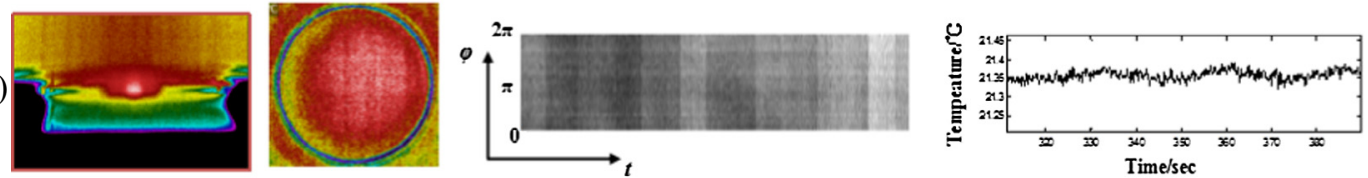

(c)
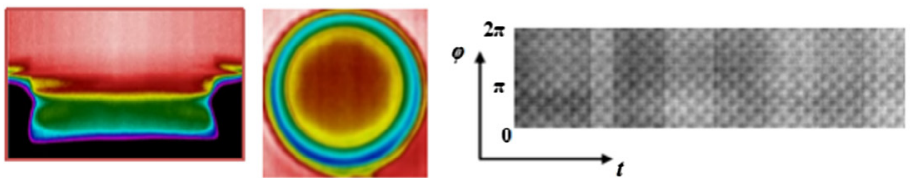

(d)
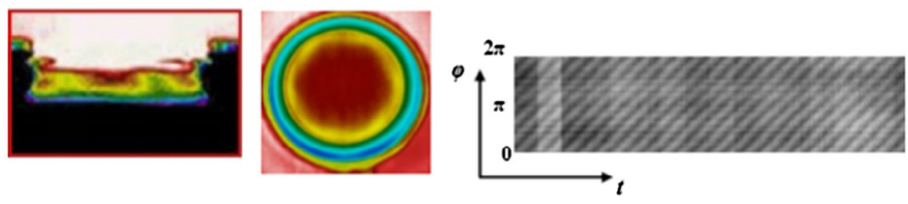

(e)
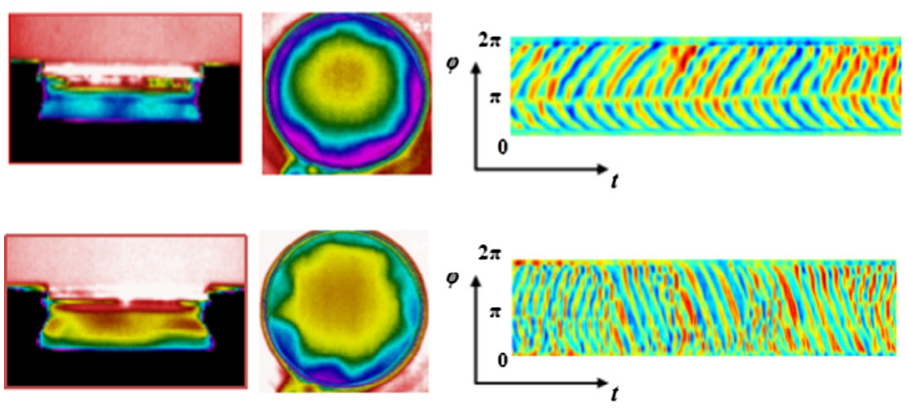
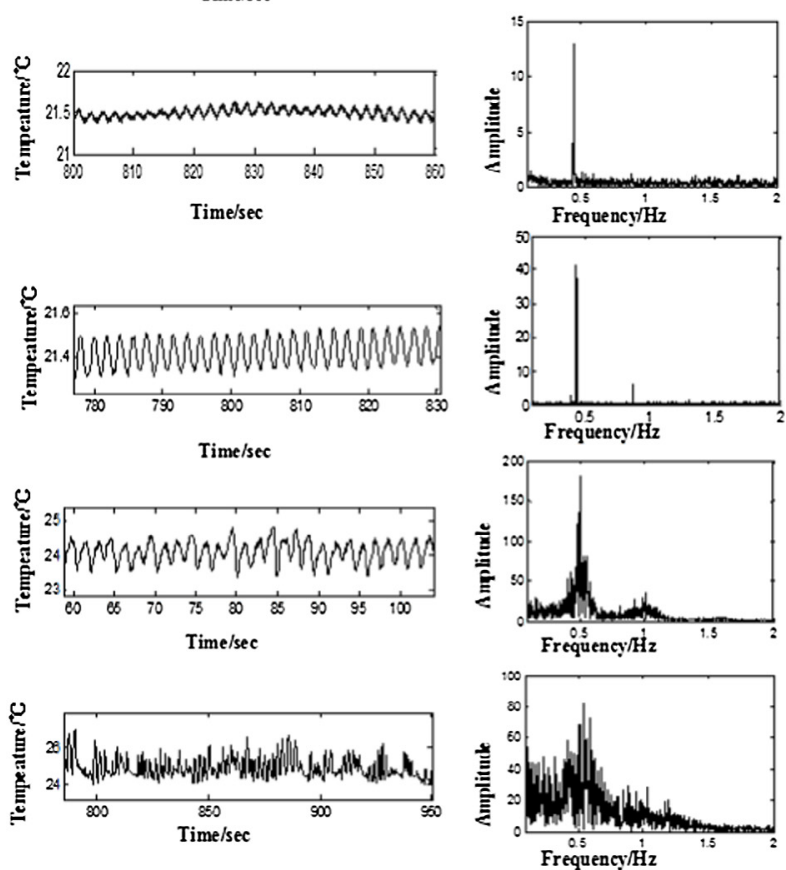

(1) Surface temperature field, (2) top plane temperature field, (3) time evolution (4), single point temperature, (5) Fourier spectrum (in log)

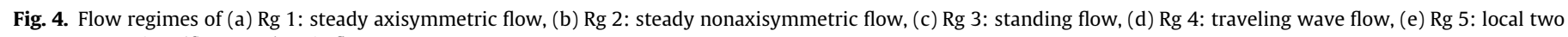
waves separation, (f) $\operatorname{Rg} 6$ : chaotic flow. 
At about $0.8 \mathrm{Ma}_{\mathrm{c}}$, the off-center state $\operatorname{Rg} 2$ is reached, where the flow remains steady without circumferential motion yet is asymmetric, and the center of temperature field deviates progressively from the center of the upper rod. At this point, the free surface temperature field shows decreased strip temperature distribution from the cold to the hot side, thus simultaneously indicating that the fluid circle in the radial cylindrical axis $(r-z)$ plane and single point temperature with the same curvature change with time (as shown in Fig. 4(b)). In addition, the observation by thermocouples at the five different azimuths in the bridge proves that the temperature has no obvious oscillation, neglecting small external environmental disturbance.

Once above $M a_{\mathrm{c}}$ in $\mathrm{Rg}$ 3, at the early stage of the oscillation, different from the Japanese KIBO results [23,24], it is found that the field incipiently exhibits pulsating flow of which peaks and troughs appear alternately, and single-point temperature becomes regular sinusoidal periodic oscillation, with a constant phase shift and finite-amplitude with time. The boundary between $\operatorname{Rg} 2$ and $\operatorname{Rg} 3$ has been regarded as the initial point for oscillation the field transforms from a 2D steady flow to 3D time-dependent one. In addition to the swing oscillation, the convective flow displays certain modal patterns in this regime. The fluid reveals azimuthal movement aside from the radius-cylindrical axis motion in $r-z$ plane, and the polygonal temperature region can be determined from the top view. Compared with the flow field of ground small-scale LB filled with vortex cells, the large one is more consistent with the real industrial crystal growth, in that the vortex cell is restricted in the surrounding area. That is to say, the circumferential heat transfer accelerates and temperature changes rapidly in the region from the outer boundary of the polygon to the free surface. Once the modal structure has formed in Rg 3, it is very difficult to destroy.

As a general trend, the wave is straight at the beginning of the oscillatory flow, then it becomes inclined in $\operatorname{Rg} 4$ at $1.2 M a_{\mathrm{c}}$. Through analysis of time domain on the flow, we discovered that the pulsating flow in $\operatorname{Rg} 3$ and the rotating one in $\operatorname{Rg} 4$ are the results of standing and traveling waves. The hydrothermal waves in these stages, which are developed due to the oscillatory instability of the steady flow, are described by the integer values of the azimuthal wave number. $m$ is described as the azimuthal wave number of oscillation affected by geometric parameters and temperature difference, which will later be discussed in detail.

As the bridge model is axisymmetric, the TW rotating direction is uncertain, being greatly influenced by the parallelism, concentricity, and heating uniformity of the two disks. Therefore, in $\mathrm{Rg}$ 4 the spread direction cannot be predicted, mostly in the counterclockwise rotation, and occasionally in the clockwise rotation. In addition, analyzing the azimuthally averaged solution of infrared images, we can determine the hydrothermal wave rotating direction and vortex cell structure, which coincides with the numerical calculation results of Watanabe [25] and Xun [26]. One can see that the temperature pattern consists of $2 \mathrm{~m}$ sectors, which, like $2 \mathrm{~m}$ pieces of a cake, is separated by sharp radial stripes formed by close isotherm [27]. The two neighboring cells respectively represent cold and heat regions, and are seen in pairs (Fig. 5).

With further increased Marangoni number, after the first emergence of SW in $\operatorname{Rg} 3$ and TW in $\operatorname{Rg} 4$, the flow enters a long-term (a)
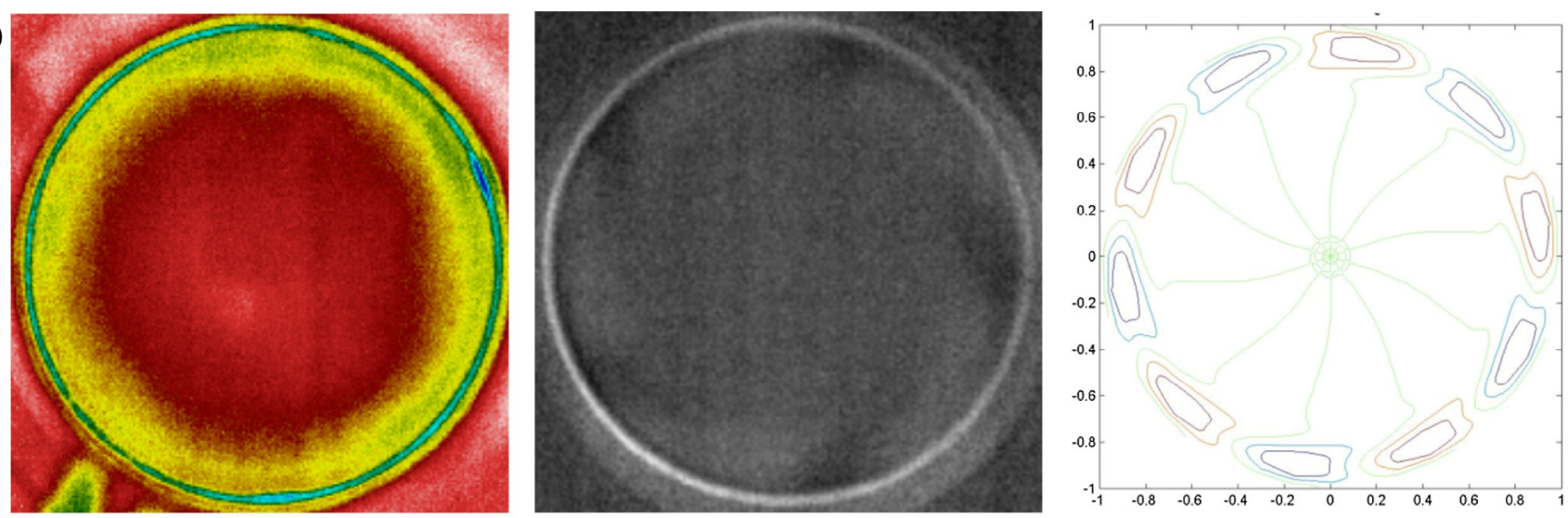

(b)
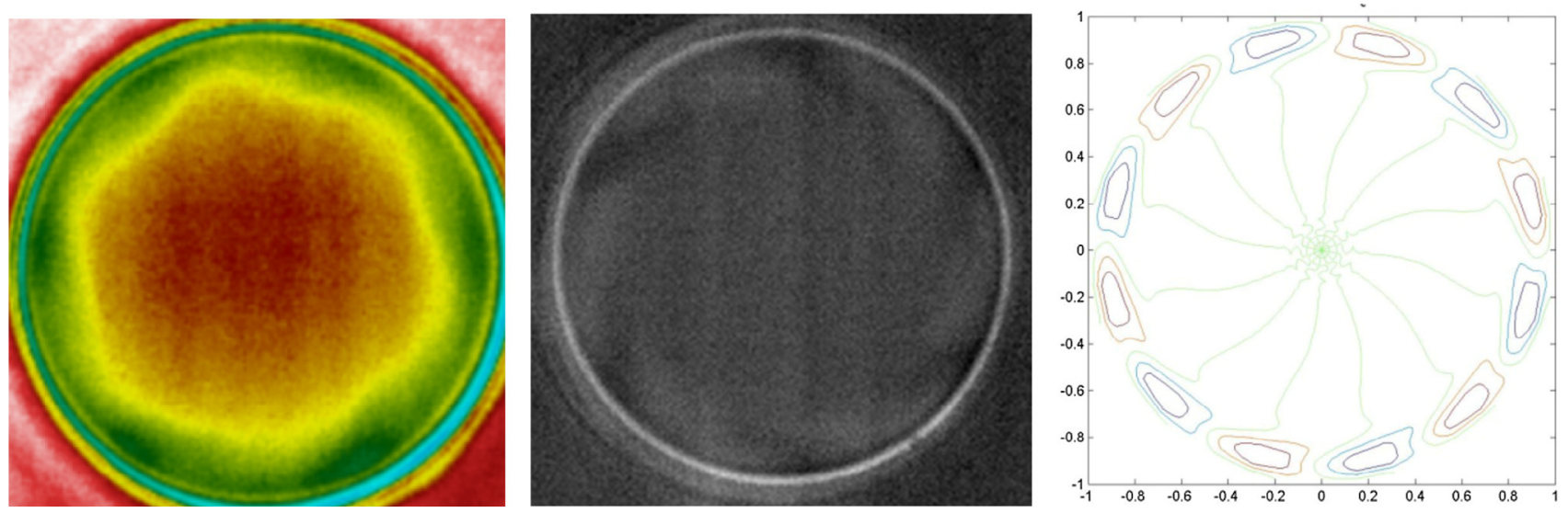

(1) Original images,

(2) the azimuthally averaged solution, (3) numerical calculation by Xun

Fig. 5. Top view of the modal structures of the temperature field: (a) $m=5$; (b) $m=6$. 
intermediate range with combinations of those two waves. At first, the main waveform is TW, then at about $1.7 M a_{\mathrm{c}}$, it increases affiliated with the standing one demonstrated in Fig. 5(a). The difference of the modal structures between our results and previous works is likely due to the diameter, aspect ratio of LB, heating rate or working fluid.

At about $1.9 M a_{\mathrm{c}}$, a novel type of flow state within the separation of local two waves is found in Rg 5. Up to this stage, the Fourier spectrum corresponds to a periodic motion with a fundamental linearly growing frequency and its harmonic ones near the instability threshold [28]. In this period, the surface-temperature distribution mostly loses the periodic. An analysis of its properties reveals that it behaves like "TW"s, which separate successively from certain points of the circumference and drift in the opposite direction, one counter-clockwise and the other clockwise without intersection. Starting from almost four axisymmetric cells, they transform into similar TWs. The cells at the lower part of the unrolled interface are almost straight, while the wave in the upper part becomes inclined. The most remarkable state is exhibited in Fig. 4(e), in which the cells near the upper and lower walls have different inclination angles. Since two energy waves may vary, the separation point is not fixed and shifts over time, and the phase difference remains constant until the separation point moves. For the first time we report this novel flow organization, and in the future further study must be carried on a greater number of its characteristics.

Above $2.1 M a_{\mathrm{c}}$ in the far supercritical area, in $\operatorname{Rg} 6$ the flow becomes obviously confused. Its power spectrum becomes rather broadened, and the basic frequency is almost buried in the continuous ones. The isotherm of the free surface becomes so fragmented that it is not possible to identify any well-organized flow pattern in this regime. The flow beyond this state could not be obtained, as a further temperature difference could not be achieved in this system.

The study of conditions for generating standing and traveling waves in an LB has attracted extensive interest among researchers. Leypoldt et al. [29] have pointed out that the stable solution for $\operatorname{Pr}=4$ is traveling waves, by computing the coefficients in Ginzburg-Ladau equations for the nonlinear evolution of hydrothermal waves. By analyzing the amplitude equation, Shevtsova [10] reported that, in addition to a set of characteristic parameters determining the structure of the oscillatory flow, the type of measured hydrothermal waves may also depend upon the initial perturbations added to the dynamical system. The temperature provided weak disturbance to the fluid instability, and the SW is observed. For the case of strong external nonsymmetrical disturbances supplied by the increasing temperature difference, the system, being at point "b" (as shown in Fig. 7), can change the trajectory and transform from SW to TW. In addition, point "b" is located not far away from the final stable, and the perturbations were strong enough to maintain the TW for a long waiting time.

Muehlner et al. [30] found that it was a feature of the oscillatory behavior of liquid with large Pr numbers that the SW was generated at the threshold of instability, although the initial perturbation introduced into the bridge was nonsymmetrical. However, taking many factors into account, the initial perturbations in the ground, microgravity experiments and computations are different. It is found the mode of oscillation changed from the rotating to standing mode under larger Marangoni number condition in KIBO [17]. Having a statistical origin, it is impossible for us to accurately forecast the type, or even the amplitude, of the perturbations in natural experiments.

As is widely known, wave motion is a continuous process of energy concentration and dissipation. There are two waves propagating in the opposite direction in the temperature field during the transition process. If their energies with the same frequency are equal, they form a standing wave. Once one of them gathers more energy than the other, the flow will transform into a traveling wave. When the energies of the two waves are almost the same and continue to increase, they will spread in opposite directions from a certain point. When the flow field has gathered enough energy, the wave energy will drastically change and the field will enter an unstable disordered state.

\subsection{Flow field structure}

Thermocapillary convection is caused by uneven surface tension, while at the same time convection also affects the temperature distribution. To provide a reference for industrial crystal growth, theoretical and experimental studies on the identification of the flow regimes from stability to instability in LBs have been

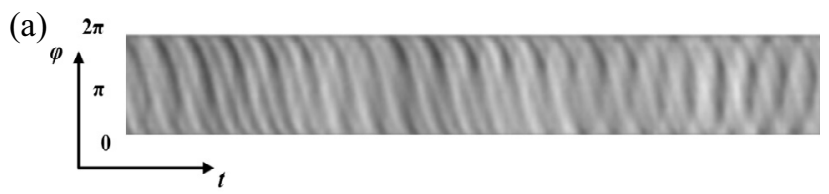

(b)

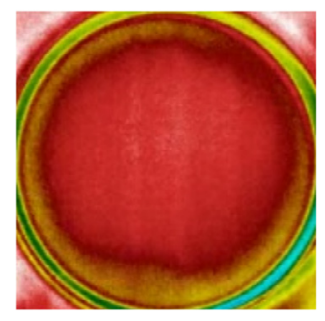

$t=\mathrm{T} / 2$

(c)

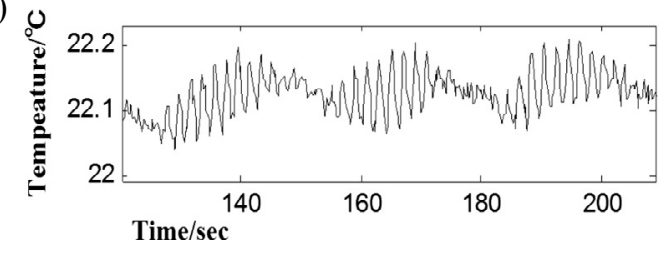

Fig. 6. Intermediate range in $\mathrm{Rg} 4$ : (a) time evolution, (b) top view temperature field, (c) single point temperature.

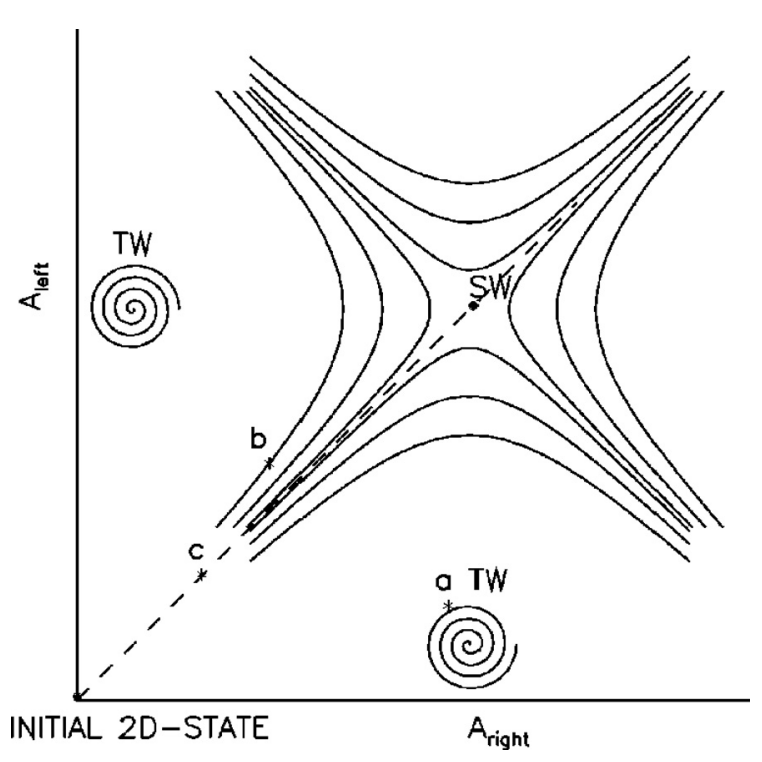

Fig. 7. Schematic phase plane. The stable state is the traveling wave $\sim$ TW. 
performed over the past several decades. Steady-state axisymmetric thermocapillary convection was first destabilized to a steady non-axisymmetric flow, then to oscillatory flow under small Prandtl number conditions [3], while for the large Prandtl number of LBs the flow directly lost its instability to the oscillation flow. Hu et al. [31,4] found that there were two similar transitions in high Prandtl numbers, similar to the one in a cylindrical LB of low Prandtl number given by LSA.

To explain the instability mechanism, $\mathrm{Hu}[32,14]$ first proposed the concept that there were two transition processes before oscillation in the thermocapillary convection of LBs with large Prandtl number. However, this problem has not been deeply studied in Japanese KIBO space experiments, thus we verify this theory on the ground, and hope that it can be observed in TG-2 space experiments.

Analyzing the flow structure under different Marangoni numbers is conducive to revealing the thermocapillary convection structure and transition process in LBs. Fig. 6 is the velocity vector diagram by cross-correlation calculation, in which the $\mathrm{Ar}$ is 0.18 and $V r$ is 0.9 . Due to the different refractive indexes of silicone

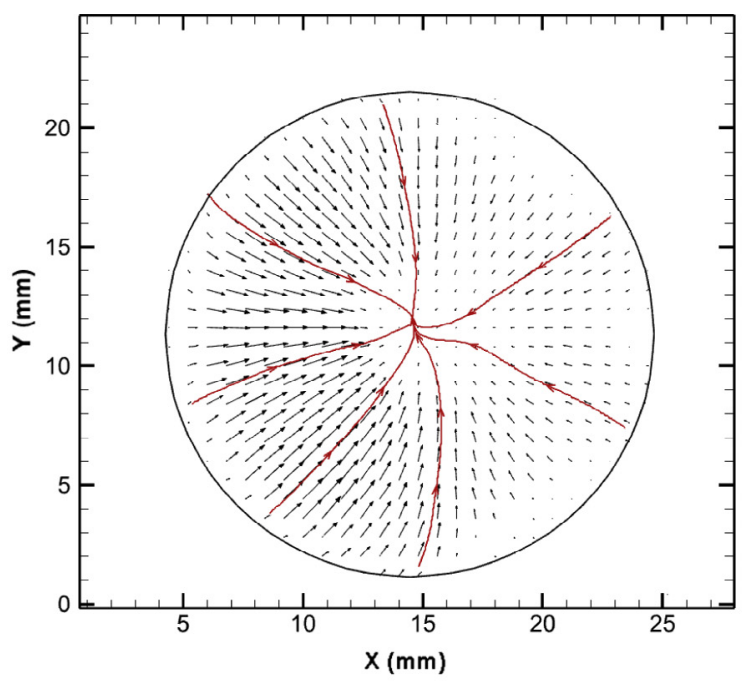

(a) Steady axisymmetric flow field

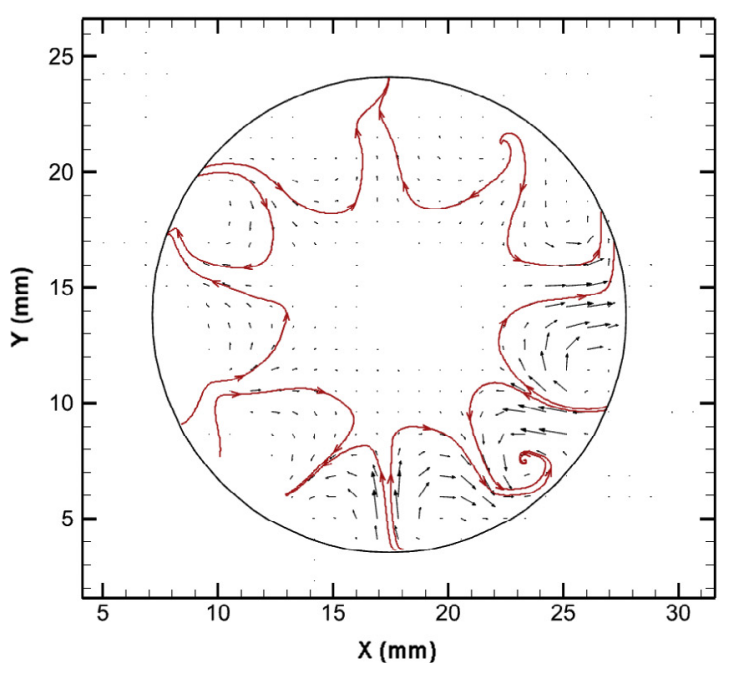

$\mathrm{t}=0$ oil and air, the light sheet is compressed at the outlet of the edge in the measured cross-section. Speed cannot be acquired in the outer edge area due to the absence of light. On account of the symmetry of motion, the entire flow field can be obtained by the extension of the half observed part. In order to better understand the structure of the flow field, we obtained the fluid trajectory by the path line apart from the velocity vector.

Under the joint action of buoyancy and surface tension, the flow field was shown to be axisymmetric and stable without an apparent vortex cell structure when the $M a$ was less than $0.8 M a_{c}$, and the velocity accelerated up to several $\mathrm{mm}$ per second. Consistent with the temperature field consequences, the stream is a 2D planar motion limited to the $r-z$ plane. The velocity field distribution is uniform, and the directions of the particles velocity in the same radial are the same as in the flow field. Furthermore, all of the path lines are relatively flat and converge at the center of the circle. The increasing temperature difference shows a widened surface tension gradient, and once it reaches $\mathrm{Rg} 2$, the field becomes nonaxisymmetric with $m=1$, so that the center of the flow deviates from that of the hot rod, although the path lines are not curled. This

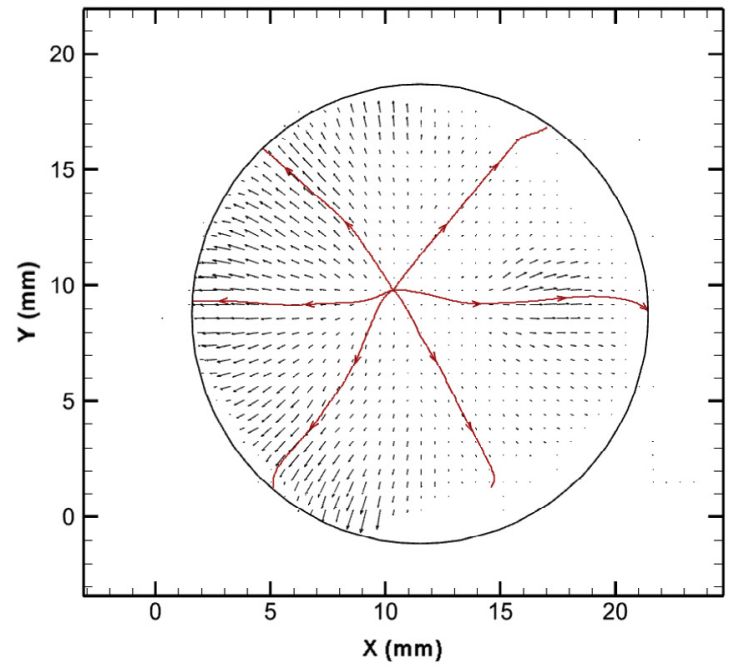

(b)Steady non-axisymmetric flow field

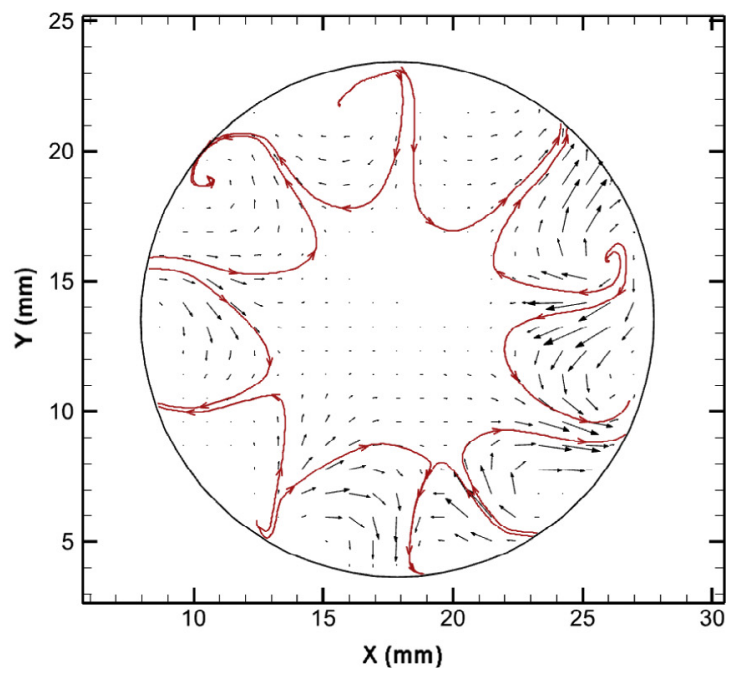

$\mathrm{t}=\mathrm{T} / 2$

(c) Oscillatory state

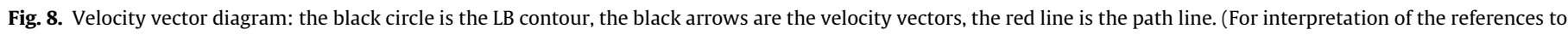
color in this figure legend, the reader is referred to the web version of this article.) 
stationary process is regarded as the first transition where the axisymmetric flow field translates into the non-axisymmetric one.

However, above $M a_{c}$, the flow in the bridge becomes unstable, and unlike the central part movement, the fluid at the edge area possesses circumferential velocity components. As shown in Fig. 8(c), the movement direction of the vortex cell can be observed more intuitively, so that five pairs of vortex cells structure appear in the field. Quoting the explanation of Schwabe [33], there is a temperature maximum or minimum value between two adjacent vortex cells. The cell directional flow induces hydrothermal wave motion, which is the traveling wave observed in the temperature field by IR. This process is known as the second transition process. The onset of bifurcation for the case of large Prandtl number could be significantly induced by both the hydrodynamic and thermal effects, not only by the hydrodynamic effect, as suggested for low Prandtl number. Furthermore, the conclusion of the appearance of the bifurcation agrees with the results of linear stability analysis given by Chen et al. [31] and Tang et al. [4].

\subsection{Volume ratio effect and modal structures of oscillatory flow}

The flow field in the LB shows a transition from a 2D steady flow to a 3D time-dependent one once the $M a$ exceeds the critical values. The critical condition in terms of Marangoni number as a function of the aspect ratio $A r$ has been explored for many years. However, according to the theory of linear stability analysis proposed by Wanschura et al. [34], the critical Marangoni number slightly relies on the $A r$ when it is lower than about 0.2 that the maximum $\mathrm{Ar}$ of large-scale LB could realize due to the gravity and shape deformation. The inherent dependency of the critical value upon the aspect ratio may not be as important as we had expected, as it cannot solely explain the large dependence of $M a_{\mathrm{c}}$ upon the geometric parameters. Moreover, the shape of the interface will be greatly determined by the liquid volume, thus we concentrate on the impact of the volume ratio on the critical conditions (see Fig. 9).

Different from the idealistic shape without deformation generated in microgravity [17] and the small deformation in the small scale one in $1 \mathrm{~g}$, the deformation in large-scale liquid is very obvious. With the increase of $V r$, the column neck diameter increases, where it is a concave free surface, while in contrast near the lower disk it is a convex free surface.

Combined with the thermocouples and infrared results, Fig. 10 exhibits the findings for a wide range of LB volume ratio $\mathrm{Vr}$ from 0.60 to 1.0 . The critical Marangoni number is plotted versus $V r$, which has strong nonlinear characteristics. Such a trend is in strong agreement with the conclusion of the small one in $1 \mathrm{~g}$ and the large one in $\mu \mathrm{g}$, and this is first verified volume ratio effect of large scale LB on the ground.

Fig. 10 includes the relationship among the critical values of the SW, TW and volume ratio of large-scale LB. As the volume ratio increases, the critical oscillation curves are divided into two branches, namely "fat" and "slender" bridges. The typical thermocapillary convection of the half floating zone LB contains the stability, instability and clearance regions [14], and the field in $\operatorname{Rg} 1$ is 2D steady flow consisting of $\operatorname{Rg} 1$ and $\operatorname{Rg} 2$ as mentioned above. Up to
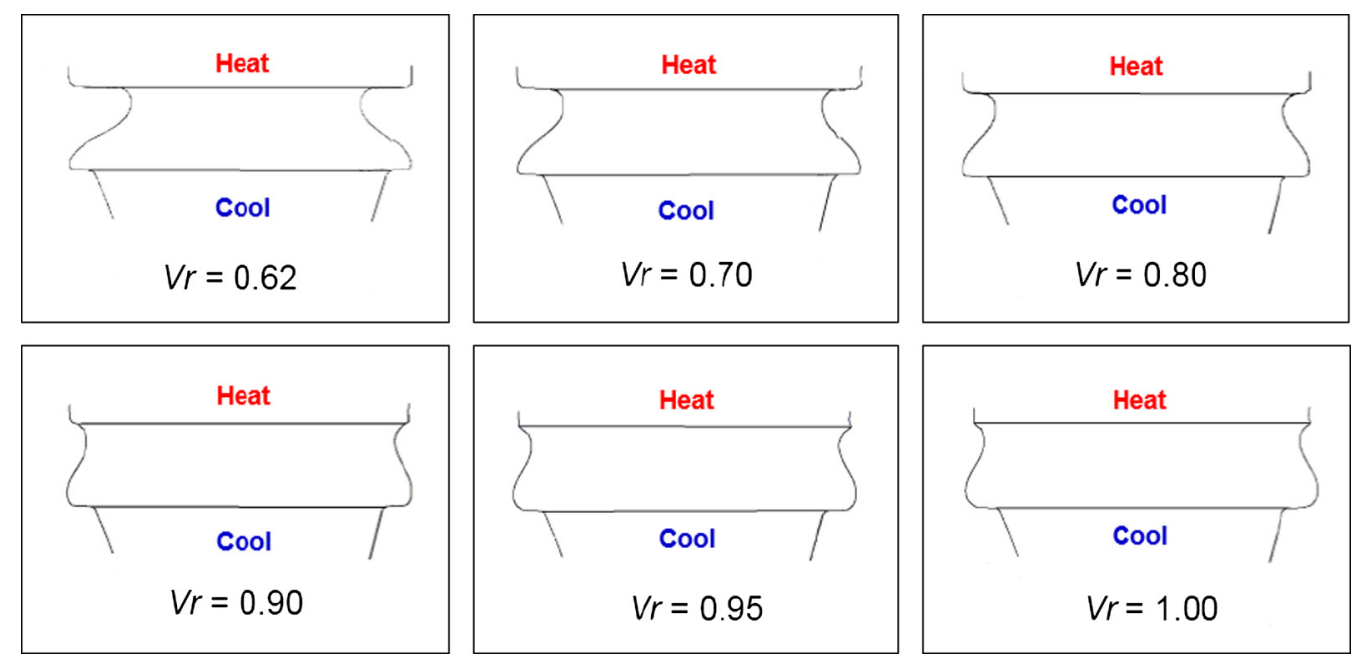

Fig. 9. Shapes of LBs for different $V r$ and constant $A r=0.2$ (the free surface topography of the LB is obtained by edge extraction).

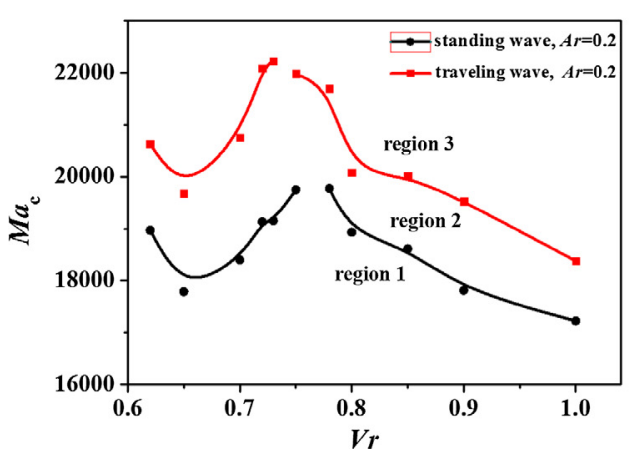

(a) Volume ratio effect of large scale LB

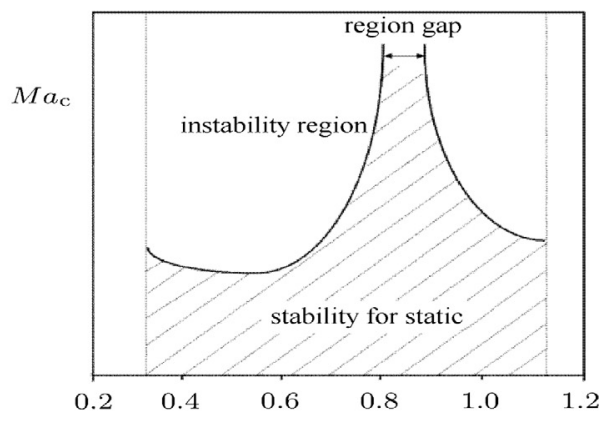

(b) Volume ratio effect of small scale LB

Fig. 10. Typical marginal curves depending on liquid bridge volume for the onset of oscillatory thermocapillary convection. 
$\operatorname{Rg} 3$, the flow fails to maintain stability and becomes an instability region, corresponding to the $\mathrm{Rg} 3$ within the SW type pulsating flow emerging in the flow field. $\operatorname{Rg} 4$ and the other regions successively appear in this region. It may be observed there is a peak around $V r=0.75$, indicating that the Marangoni convection is most stabilized around this volume ratio for both standing and (a)

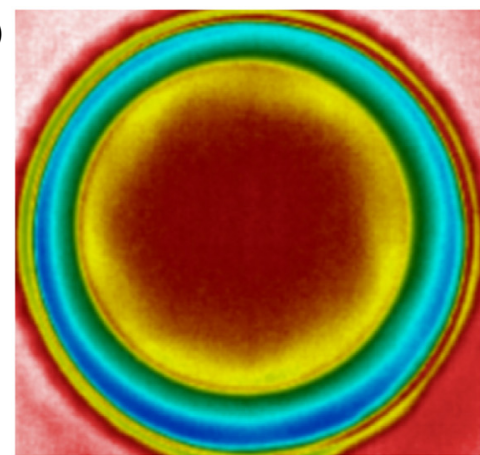

$A r=0.15, V r=0.57$

(b)

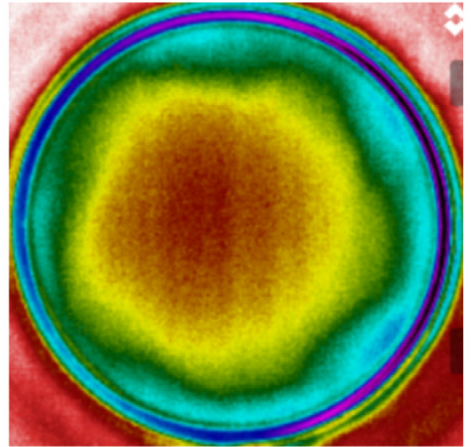

$A r=0.17, V r=0.78$

(c)

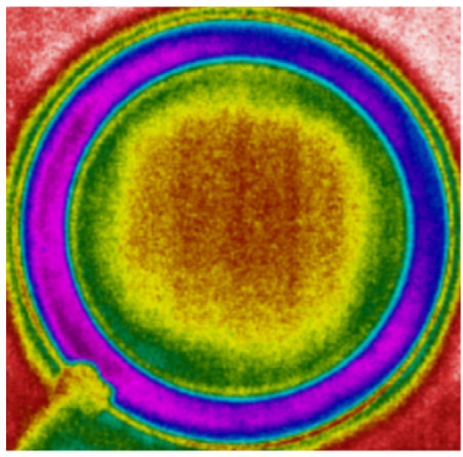

$A r=0.17, V r=0.56$

(d)

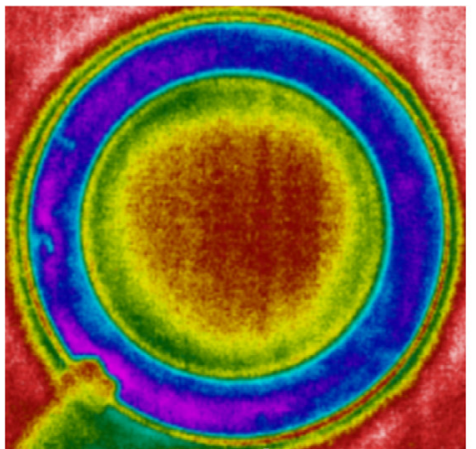

$A r=0.20, V r=0.54$

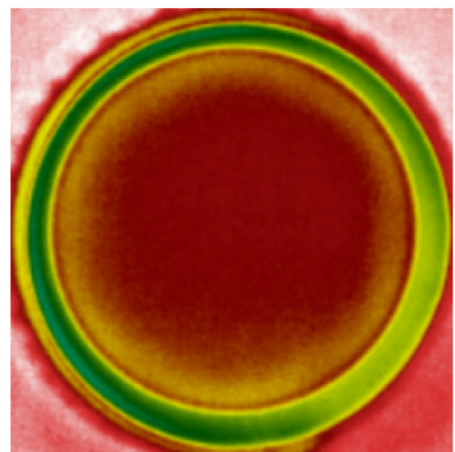

$A r=0.15, V r=0.69$

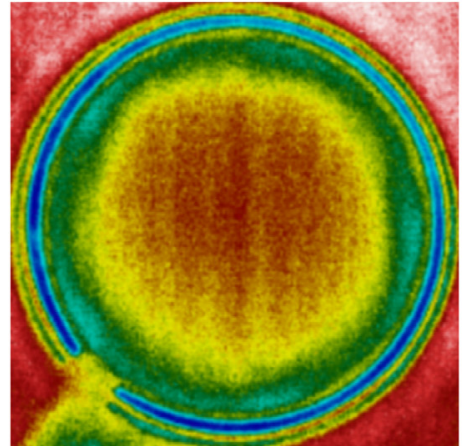

$A r=0.18, V r=0.82$

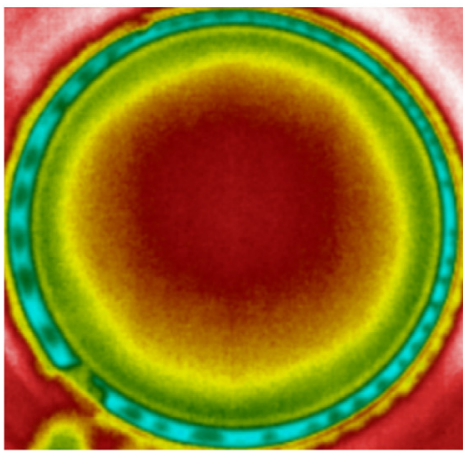

$A r=0.20, V r=0.75$

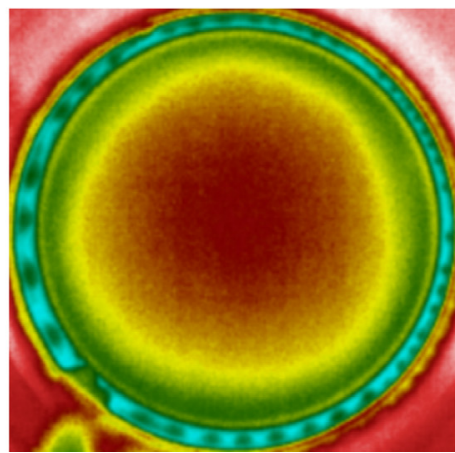

$A r=0.20, V r=0.75$

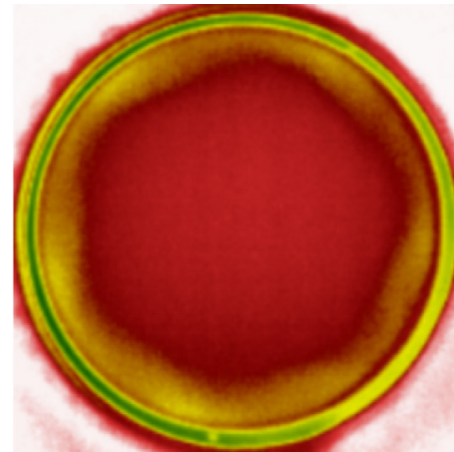

$A r=0.15, V r=0.82$

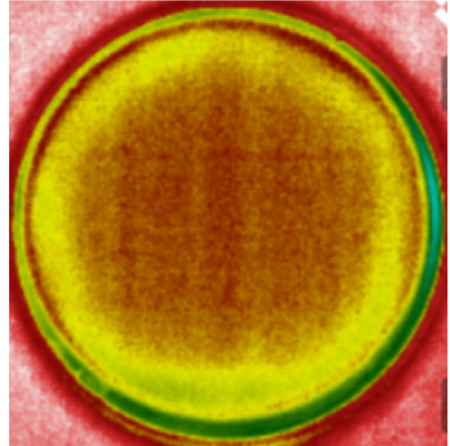

$A r=0.20, V r=0.91$

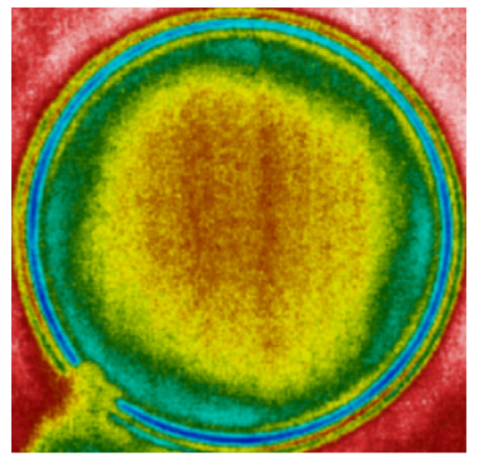

$A r=0.20, V r=0.89$

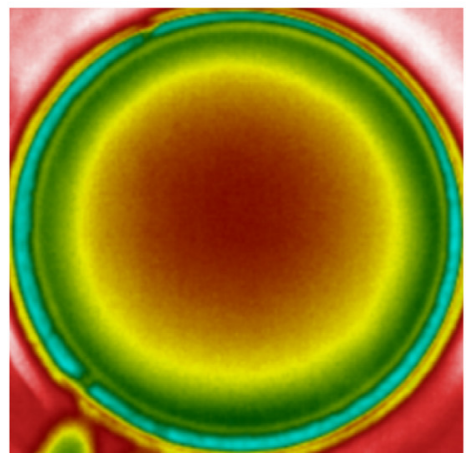

$A r=0.20, V r=0.89$

Fig. 11. Top view of modal structures of the temperature field for given aspect ratio and volume ratio; (a) modal wave number $m=6$, (b) $m=5$, (c) $m=4$, (d) $m=3$. 
traveling waves, which is known as the clearance region. In this region, the flow field oscillation requires higher $\Delta T$. In contrast, the data are smaller than the $V r=0.8-0.9$ studied by $\mathrm{Hu}$ et al. [14] and Masud et al. [35] in their $1 \mathrm{~g}$ small-scale LB experiments, and numerically by Chen and $\mathrm{Hu}$ et al. [7], but larger than the $V r=0.53$ observed in KIBO $(A r=0.5, D=30 \mathrm{~mm}, 20 \mathrm{cSt}$ silicone oil). In addition to gravity, this difference likely occurs as a result of the difference in the size of the LB, as well as that in Pr number. Based on industrial application, the study of the volume ratio effect is beneficial to selecting a suitable volume ratio within a certain diameter and aspect ratio, to delay or avoid oscillation in LBs.

As mentioned in the small-scale LB experiments, the two branches are associated with different flow structures. When the ratio $>0.75$, the $M a_{c}$ decreases noticeably as the $V r$ increases. This

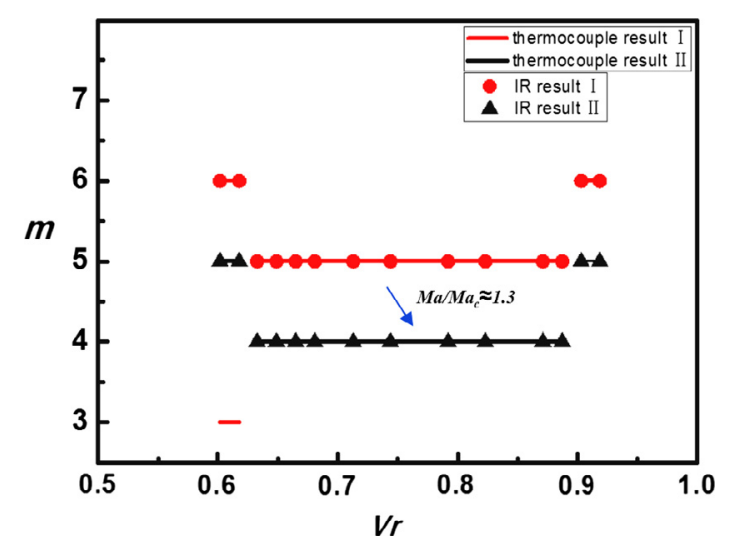

Fig. 12. Typical marginal mode number curves of thermocouples and IR results for $A r=0.2$, with $V r$ from $0.60-0.93$. decrease is due to the fact that the average column diameter increases, and consequently the overall flow is measured to speed up for a given $\Delta T$, an effect which is similar to that caused by decreasing $\operatorname{Ar}$ [35].

The flow structures have been visualized and quantified by means of infrared temperature distribution with different geometric parameters (Fig. 11). Compared with the traditional method of particles accumulating in a coherent structure (PAS), there is no time delay caused by particle accumulation and adsorption in the IR temperature measurement. By this means, errors can be avoided by observing the IR results in real-time.

The field demonstrating different wave numbers with different Ars has already been proven by Preisser et al. [36] and Ueno et al. [37]. Furthermore, they also pointed out that the value of $m \cdot A r$ remained roughly at $1-1.1$. However, subsequent researches have confirmed that $m$ and $A r$ do not strictly hold for this empirical relation. As the critical temperature difference is greatly affected by volume ratio, the wave number also can be influenced by this parameter rather than be determined merely by aspect ratio. Similar to the PAS results, the void region appears near the axis of the LB when the flow enters the oscillatory state, and the field exhibits particular shapes depending on the azimuthal mode number $m$. For the given conditions, two or three neighboring mode numbers appear, and it is not possible to anticipate which modal structure first emerges at the onset of the oscillation. Fig. 12 demonstrates that the variation of the wave number with the varied volume ratio for $A r$ is 0.2 . The critical wave number may be 4 or 5 for the volume ratios from 0.62 to 0.9 , and 5 or 6 for the rest, when $V r$ is $0.60-0.62$. The growth of the wave number is caused by a reduction in the effective height, equivalent to the reduction in $\mathrm{Ar}$.

However, for larger Marangoni number, at about $1.3 M a_{c}$, the wave number decreases gradually, and the product of $m \cdot A r$ tends to be constant. As an important energy source of the entire flow

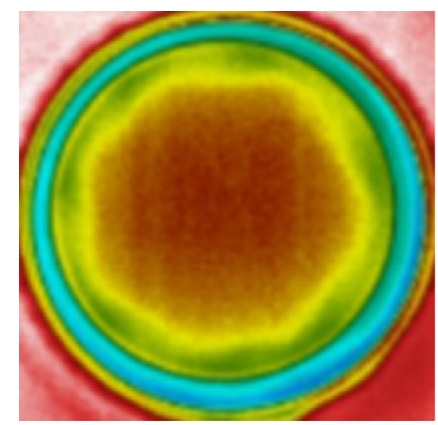

$m=7$

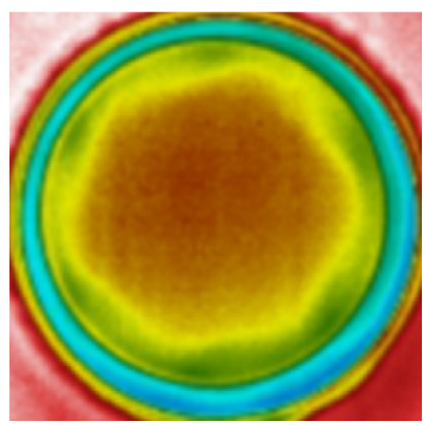

$m=6$

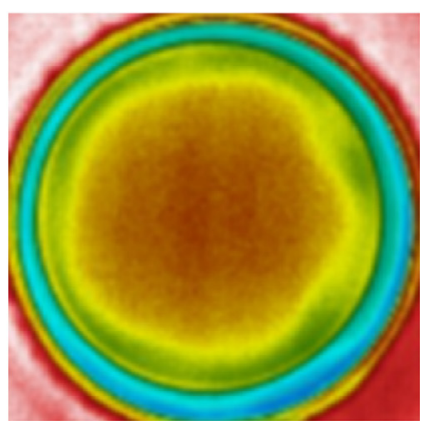

$m=5$

0-1 $\min :$

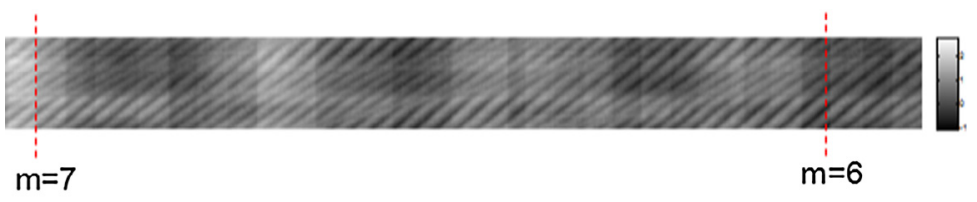

4-5min:

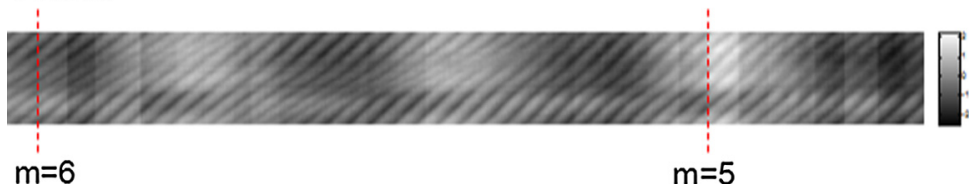

Fig. 13. Mode transformation with increasing temperature difference. 
system, the flow mode is sensitive to the temperature difference [38]. At the beginning of the oscillation, the flow is axisymmetric, the temperature of single point may change with the increasing temperature, then reaches a stable state again after the transition. To prove this, in Fig. 13 we set the circumferential time evolution of the infrared images for $A r$ as 0.16 and that of $V r$ as 0.65 . The initial critical mode is 7 , then it reduces to 6 with increasing temperature differences in one minute, and finally the wave number stabilizes at 5 with the temperature difference increasing $1.5^{\circ} \mathrm{C}$.

At the same time, we also calculate the wave number $m$ in $\mathrm{Rg} 4$ using the inversion transformation of the phase differences among the five thermocouples. Note that the inversion results are in strong agreement with the infrared data in Fig. 12, and the difference between $m \cdot A r$ may be due to the ambient gas or gravity which causes the deformation of the surface and buoyant convection. Lappa et al. [39] numerically studied the fact that heating from above and below the bridge can cause a difference in $m \cdot A r$ under normal gravity. Koichi et al. [40] reported that the product of the above two parameters is about 0.7 in microgravity, by using linear stability analyses (LSA). These results, including those of the present study, suggest the need for further research regarding the effects of conditions on the modal structures of LBs.

\subsection{Experimental uncertainty analysis}

The inevitable errors in the experimental measurement can influence the accuracy of the final results. The uncertainty analysis method applied in this work is one of the most widely used error analysis methods by which to calculate the uncertainty of the experimental parameters. Based on the work of Li [41], when the error of the physical properties is neglected, the uncertainty of the Marangoni number can be simplified as follows:

$U_{M a}=\left[\left(\frac{\partial M a}{\partial T_{\text {heat }}} U_{\text {heat }}\right)^{2}+\left(\frac{\partial M a}{\partial T_{\text {cool }}} U_{\text {cool }}\right)^{2}+\left(\frac{\partial M a}{\partial H} U_{H}\right)^{2}\right]^{1 / 2}$

Where the $T_{\text {heat }}$ and $T_{\text {cool }}$ respectively represent the temperature imposed on the upper and lower disks. The uncertainty of the volume ratio $(V r)$ caused by liquid injection, and the diameter and height of the bridge are calculated as follows:

$U_{V r}=\left[\left(\frac{\partial V r}{\partial h} U_{h}\right)^{2}+\left(\frac{\partial V r}{\partial r} U_{r}\right)^{2}+\left(\frac{\partial V r}{\partial H} U_{H}\right)^{2}\right]^{1 / 2}$

Coleman et al. [42] pointed that $95 \%$ of the random standard uncertainty related to the readability can be regarded as $50 \%$ of the minimum scale division. For the digital instrumentation, we take the smallest division value as the instrument error. Therefore, the random standard uncertainties in the temperature, height, diameter, and PI motor-driven injection are $0.05^{\circ} \mathrm{C}, 0.01 \mathrm{~mm}$, $0.05 \mathrm{~mm}$ and $3.5 \times 10^{-6} \mathrm{~mm}$, respectively.

From the calculated results, it is found that the relative combined standard uncertainty of the thermocapillary Marangoni number ranges from 0.48 to $0.64 \%$. The maximum evaporation in one experiment is far less than $10 \mu \mathrm{L}$, thus the relative combined standard uncertainties of the final volume ratio can change range from 0.67 to $0.70 \%$.

\section{Summary and conclusions}

This paper reports the experimental results regarding the instability of buoyant-thermocapillary convection in large-scale liquid bridge with large Prandtl number under normal gravity conditions. The internal flow structure of the large-scale liquid bridge is complicated, thus the research regarding it can promote the understanding of the nonlinear characteristics, flow stability and bifurcation transition of thermocapillary convection. In addition to single point temperature measurement by thermocouples, the IR camera and PIV are applied respectively to measure the temperature field and velocity field of the bridge. Far beyond the critical condition is experimentally investigated, the flow mode, volume ratio and transition process are studied in detail.

The changes of the flow structure from steady state to chaos are observed through the free surface and cross-section temperature distribution measurements with IR camera. With the increasing temperature difference, the nonuniformity of temperature gradient distribution on the free surface is shown to increase, i.e. there is a great temperature gradient on the hot and cold sides, while the middle temperature gradient is small. This change increases the possibility of hot and cold spots appearing in liquid bridges, which leads to the instability of the internal flow. It is shown that the flow field may change gradually from the steady axisymmetric state to chaos, and the increased temperature difference introduces the initial perturbation to the flow field, resulting in a standing wave state. However, the standing wave is not stable, thus further increasing the temperature difference, the external factor interference is expanded, and the flow field enters the traveling wave state after the transition. Due to the fact that the traveling wave is in a relatively stable motion state, though there may be small disturbance, the flow field can still maintain a traveling wave state for a long period of time. After two waves separate from one certain point, the flow field finally enters the chaotic state due to the joint effect of the buoyancy and surface tension.

The effects of the liquid bridge shape on the instability of the LB are studied by changing the volume ratio, $V r$, which is defined as the ratio of the volume of the LB and the volume of the cylindrical gap between the upper and lower disks. The intimal oscillatory data taken from large-scale LBs indicate a significant effect of gravity on the instability. It is demonstrated that the critical temperature difference curve of the oscillation shows a nonlinear characteristic with increasing $V r$, and the convection is most stabilized at certain Vrs. However, due to gravity, the free surface of the LB is greatly deformed, being affected by the Prandtl number and minimum diameter of the LB, and certain Vrs are smaller than those observed in the previous small-scale LBs, but larger than the large ones in $\mu \mathrm{g}$.

It is demonstrated that, for the parametric research of problems with flow mode, the choice of the critical wave number is random, and basically follows the relationship between the wave number and aspect ratio, namely $m \cdot A r \approx 1$. At the same time, it is found that the flow oscillation mode is sensitive to the temperature difference in the traveling wave region far from the critical point, the wave number decreases as the natural convection of internal, and the external LB increases as the temperature difference increases.

\section{Acknowledgments}

This research study was funded by the China Manned Space Engineering program (TG-2), the National Natural Science Foundation of China (11372328), and the Strategic Priority Research Program on Space Science, Chinese Academy of Sciences: SJ-10 Recoverable Scientific Experiment Satellite (XDA04020405 and XDA04020202-05).

\section{References}

[1] Wenrui Hu, Zemei Tang, Floating Zone Convection in Crystal Growth Modeling, Science Press, Beijing, New York, 2003, pp. 8-12.

[2] Qi Kang, Li Duan, Li Zhang, Yongli Yin, Jingsong Yang, Hu Wenrui, Thermocapillary convection experiment facility of an open cylindrical annuli for SJ-10 satellite, Microgravity Sci. Technol. 2 (28) (2016) 123-132.

[3] M. Levenstam, G. Amberg, Hydrodynamic instabilities of thermocapillary flow in half zone, Fluid Mech. 297 (1995) 357-372. 
[4] Z.M. Tang, W.R. Hu, N. Imaishi, Two bifurcation transitions of the floating half zone convection, Int. J. Heat Mass Transfer 44 (2001) 1299-1307.

[5] K. Li, Satoshi Matsumoto, Nobuyuki Imaishi, Wen-Rui Hu, Marangoni flow in floating half zone of molten tin, Int. J. Heat Mass Transfer 83 (2015) 575-585.

[6] K. Li, B. Xun, N. Imaishi, S. Yoda, W.R. Hu, Thermocapillary flows in liquid bridges of molten tin with small aspect ratios, Int. J. Heat Fluid Flow 29 (2008) 1190-1196.

[7] Q.S. Chen, W.R. Hu, Influence of liquid bridge volume on instability of floating half-zone convection, Heat Mass Transfer 41 (1998) 825-837.

[8] JJ. Xu, S.H. Davis, Convective thermocapillary instabilities in liquid bridges, Phys. Fluids 27 (1984) 1102-1107.

[9] Z. Zeng, H. Mizuseki, K. Higashino, Y. Kawazoe, Direct numerical simulation of oscillatory Marangoni convection in cylindrical liquid bridges, J. Cryst. Growth 204 (1999) 395-404.

[10] V.M. Shevtsova, D.E. Melnikov, J.C. Legros, Three-dimensional simulations of hydrodynamic instability in liquid bridges: Influence of temperaturedependent viscosity, Phys. Fluids 13 (2001) 2851-2865.

[11] R. Monti, M. Lappa, R. Savino, Flight results of Marangoni flow instability in liquid bridge, IAF, 1999, J. 3. 01.

[12] F. Preisser, D. Schwabe, A. Scharmann, Steady and oscillatory thermocapillary convection in liquid columns with free cylindrical surface, Fluid Mech. 126 (1983) 545-567.

[13] Masato Sakurai, Naoya Ohishi, Akira Hiratab, Oscillatory thermocapillary convection in a liquid bridge: Part 1-1g experiments, J. Cryst. Growth 308 (2007) 352-359.

[14] W.R. Hu, J.Z. Shu, R. Zhou, Z.M. Tang, Influence of liquid bridge volume on the onset of oscillation in floating-zone convection I. Experiment, J. Crystal Growth 142 (1994) 379-384.

[15] S. Frank, D. Schwabe, Temporal and spatial elements of thermocapillary convection in floating zones, Exp. Fluids 23 (1997) 234-251.

[16] D. Schwabe, Standing waves of oscillatory thermocapillary convection in floating zones under microgravity observed in the experiment MAUS G141, Adv. Space Res. 29 (2002) 651-660.

[17] T. Yano, K. Nishino, H. Kawamura, I. Ueno, S. Matsumoto, Effect of liquid bridge shape on the instability of Marangoni convection in space experiment in: 15th International Symposium on Flow Visualization, 2012, Minsk, Belarus.

[18] H. Kawamura, K. Nishino, S. Mastumoto, Report on microgravity experiments of Maragoni convection aboard international space station, Trans. ASME J. Heat Transfer 134 (2012) 031005-031018.

[19] Taishi Yano, Koichi Nishino, Hiroshi Kawamura, Ichiro Ueno, Satoshi Matsumoto, Mitsuru Ohnishi, Masato. Sakural, 3-D PTV measurement of Marangoni convection in liquid bridge in space experiment, Exp. Fluids 53 (2012) 9-20.

[20] Koichi Nishino, Taishi Yano, Hiroshi Kawamura, Satoshi Matsumoto, Ichiro Ueno, Michael K. Ermakov, Instability of thermocapillary convection in long liquid bridges of high Prandtl number fluids in microgravity, J. Crystal Growth 420 (2015) 57-63.

[21] Shin-Etsu, Chemical Co., Ltd., Silicone Fluid (product catalogue), 2004, pp. 1213.

[22] William Feller, An Introduction to Probability Theory and its Applications, vol. I, John Wiley \& Sons Inc, New York, 1950, pp. 164-187.

[23] D.E. Melnikov, V. Shevtsova, T. Yano, K. Nishino, Modeling of the experiments on the Marangoni convection in liquid bridges in weightlessness for a wide range of aspect ratios, Int. J. Heat Mass Transfer 87 (2015) 119-127.

[24] Taishi Yano, Koichi Nishino, Hiroshi Kawamura, Ichiro Ueno, Satoshi Matsumoto, Mitsuru Ohnishi, Masato Sakurai, 3-D PTV measurement of
Marangoni convection in liquid bridge in space experiment, Exp. Fluids 53 (2012) 9-20.

[25] T. Watanabe, The stability of a thermocapillary -buoyant flow in a liquid bridge with heat transfer through the interface, Microgravity Sci. Technol. 26 (2014) 17-28.

[26] Bo Xun, Study of the Thermocapillary Flow in Liquid Bridge and Its stability (Ph.D. Thesis), Institute of Mechanics, Chinese Academy of Sciences, Beijing, 2011 (in Chinese).

[27] Marcello Lappa, Stationary solid particle attractors in standing waves, Phys. Fluids 26 (013305) (2014) 1-12.

[28] V. Shevtsova, D.E. Melnikov, A. Nepomnyashchy, New flow regimes generated by mode coupling in buoyant-thermocapillary convection, PRL 102 (134503) (2009) 1-4.

[29] J. Leypoldt, H.C. Kuhlmann, H.J. Rath, Three-dimensional numerical simulations of thermocapillary flows in cylindrical liquid bridges, J. Fluid Mech. 414 (2000) 285-314.

[30] K.A. Muehlner, M. Schatz, V. Petrov, W.D. Mc Cormic, J.B. Swift, H.L. Swinney, Observation of helical traveling-wave convection in a liquid bridge, Phys. Fluids 9 (1997) 1850-1852.

[31] Q.S. Chen, W.R. Hu, Instability from steady and axisymmetric to steady and asymmetric floating half zone convection in a fat liquid bridge of larger Prandtl number, Chin. Phys. Lett. 6 (1999) 822-823.

[32] T.H. Han, Y. Ar, R. Zhou, W.R. Hu, Investigation on simulation model of floating half zone convection, II. Experiment, Int. J. Heat Mass Transfer 40 (1997) 26712677.

[33] D. Schwabe, A.I. Mizev, Particles of different density in thermocapillary liquid bridges under the action of traveling and standing hydrothermal waves, Eur. Phys. J. Spec. 192 (2011) 13-27.

[34] M. Wanschura, V.M. Shevtsova, H.C. Kuhlmann, H.J. Rath, Convective instability mechanisms in thermocapillary liquid bridges, Phys. Fluids 7 (912) (1995) 912-925.

[35] J. Masud, Y. Kamotani, S. Ostrach, Oscillatory thermocapillary flow in cylindrical columns of high Prandtl number fluids, J. Thermophys. Heat Transfer 11 (1) (1997) 105-111.

[36] W.R. Hu, J.Z. Shu, R. Zhou, Z.M. Tang, Influence of liquid bridge volume on the onset of oscillation in floating-zone convection I. Experiment, J. Crystal Growth 142 (1994) 379-384.

[37] Ichiro Ueno, Shiho Tanaka, Hiroshi Kawamura, Oscillatory and chaotic thermocapillary convection in a half-zone liquid bridge, Phys. Fluids 15 (2003) 408-416.

[38] Wu Yongqiang, Duan Li, Kang Qi, Ground experiments of bouyantthermocapillary convection of large scale liquid bridge with large Prandtl number, Acta. Mech. Sin. 44 (6) (2012) 981-989 (in Chinese).

[39] M. Lappa, R. Savino, R. Monti, Influence of buoyancy forces on Marangoni flow instabilities in liquid bridges, Int. J. Numer. Meth. Heat Fluid Flow 10 (7) (2000) 721-749.

[40] Koichi Nishino, Taishi Yano, Hiroshi Kawamura, Satoshi Matsumoto, Ichiro Ueno, Michael K. Ermakov, Instability of thermocapillary convection in long liquid bridges of high Prandtl number fluids in microgravity, J. Crystal Growth 420 (2015) 57-63.

[41] S.Y. Wu, J.Y. Guan, L. Xiao, Z.G. Shen, L.H. Xu, Experimental investigation on heat loss of a fully open cylindrical cavity with different boundary conditions, Exp. Thermal Fluid Sci. 45 (2013) 92-101.

[42] H.W. Coleman, W.G. Steele, Experimentation, Validation, and Uncertainty Analysis for Engineers, third ed., John Wiley \& Sons, New Jersey, 2009, pp. 128150. 